\title{
A ferroptosis-related IncRNA signature predicts prognosis in ovarian cancer patients
}

\author{
Jing Peng ${ }^{1 \wedge}$, Yan Hao ${ }^{1,2,3}$, Bihua Rao ${ }^{1}$, Zhiguo Zhang ${ }^{1,2,3} \wedge$ \\ ${ }^{1}$ Department of Obstetrics and Gynecology, Reproductive Medicine Center, The First Affiliated Hospital of Anhui Medical University, Hefei, China; \\ ${ }^{2}$ Anhui Province Key Laboratory of Reproductive Health and Genetics, Hefei, China; ${ }^{3}$ Biopreservation and Artificial Organs, Anhui Provincial \\ Engineering Research Center, Anhui Medical University, Hefei, China \\ Contributions: (I) Conception and design: J Peng, Z Zhang; (II) Administrative support: Z Zhang; (III) Provision of study materials or patients: J \\ Peng, Y Hao; (IV) Collection and assembly of data: J Peng, Y Hao; (V) Data analysis and interpretation: J Peng, B Rao; (VI) Manuscript writing: All \\ authors; (VII) Final approval of manuscript: All authors. \\ Correspondence to: Zhiguo Zhang. Department of Obstetrics and Gynecology, Reproductive Medicine Center, The First Affiliated Hospital of Anhui \\ Medical University, No. 218 Jixi Road, Hefei 230022, China. Email: zzg_100@163.com.
}

Background: Ovarian cancer (OC) is a common gynecological malignant tumor with poor prognosis. Ferroptosis is an iron-dependent modality of regulated cell death. The purpose of this study was to determine the prognostic ability of ferroptosis-related long non-coding RNAs (lncRNAs) in OC patients and construct a ferroptosis-related lncRNA prognostic model.

Methods: The Cancer Genome Atlas (TCGA) and FerrDb databases were used to collect RNA sequencing data of OC patients and ferroptosis-related genes, respectively. OC patients were randomly assigned to the training or testing set. Pearson correlation analysis was used to identify ferroptosis-related lncRNAs. Univariate Cox, Least Absolute Shrinkage and Selection Operator (LASSO), and multivariate regression analyses were performed in the training set to develop a predictive model. The model was validated in the testing set and entire set. Survival analysis, receiver operating characteristic curves, independent prognostic factor analysis, and correlation analysis with clinical features were performed to evaluate the predictive value of the model. A nomogram was established to predict the survivability of OC patients over 1, 3, and 5 years. The distribution of distinct groups was investigated using principal component analysis, and the underlying the biological functions were explored using gene set enrichment analysis.

Results: Eleven ferroptosis-related lncRNAs were determined to establish the prognostic model. Patients in the high-risk group had poor prognosis compared with the low-risk group in the training, testing and entire sets. The area under the receiver operating characteristic curve corresponding to 1-, 3-, and 5-year survival were $0.731,0.796$, and 0.805 in the training set; $0.704,0.597$, and 0.655 in the testing set; and 0.715 , 0.691 , and 0.736 , in the entire set, respectively. The risk score correlated with age and grade. The risk score was also an independent prognostic factor in OC. A nomogram with high C-index (0.68) was constructed. An intuitive observation of the principal component analysis revealed a distinction between high- and lowrisk groups, and gene set enrichment analysis indicated that cancer-related pathways were enriched in the high-risk group.

Conclusions: The signature composed of 11 ferroptosis-related lncRNAs accurately predicted the prognosis of OC patients.

Keywords: Ferroptosis; long non-coding RNAs (lncRNAs); ovarian cancer (OC); The Cancer Genome Atlas (TCGA)

Submitted Jul 02, 2021. Accepted for publication Sep 03, 2021.

doi: $10.21037 /$ tcr-21-1152

View this article at: https://dx.doi.org/10.21037/tcr-21-1152

^ ORCID: Jing Peng: 0000-0002-8895-8189; Zhiguo Zhang: 0000-0003-1483-3321. 


\section{Introduction}

Ovarian cancer (OC) is a common gynecological malignant tumor and a main cause of disease-related death in women worldwide (1). According to the latest global cancer statistics, OC was the eighth most common cancer in women and the eighth most common cause of cancer related death in women worldwide in 2020, with approximately 314,000 new cases and more than 207,000 deaths in 2020 (2). Approximately 70\% of OC patients are already at an advanced stage when diagnosed because of the lack of early diagnosis tools (3). Furthermore, over half of OC patients experience recurrence within 2 years after treatment, and the 5-year survival rate is unsatisfactory, remaining below $30 \%$ (4). Several prognostic factors for OC such as age, histological type, and FIGO stage have been identified (5). However, because of the high degree of heterogeneity of OC, developing accurate molecular biomarkers to predict treatment response and overall survival (OS) for OC patients is critical.

Regulated cell death (RCD) plays an invaluable role in the occurrence and development of tumors (6). In recent years, significant progress has been made on therapeutic measures related to RCD including autophagy, apoptosis and necrosis in OC (7-9). However, clinical outcomes in advanced OC are still unsatisfactory (3). Therefore, there is an urgent need for new therapeutic options. Ferroptosis is an iron-dependent modality of RCD that is morphologically and genetically distinct from autophagy, apoptosis and necrosis (10). The process of ferroptosis involves increased reactive oxygen species (ROS), cell membrane thickening and mitochondrial volume reduction $(11,12)$. Excessive iron-dependent oxidative stress has been shown to partially contribute to carcinogenesis (13). Furthermore, due to genetic changes and abnormal growth, cancer cells have chronically elevated levels of ROS-related oxidative stress (14). Thus, cancer cells are susceptible to ferroptosis, and therefore targeting ferroptosis has been considered a promising therapeutic option, especially for malignancies that are resistant to traditional therapies $(15,16)$. Multiple studies have confirmed that ferroptosis plays an important role in OC. For example, Wang et al. reported that platinum-tolerant OC cells are highly susceptible to ferroptosis (17). Furthermore, an overexpressed p53 could contribute to ferroptosis in OC cells (18) and erastin works synergistically with cisplatin to inhibit the growth of OC cell by a ROS-mediated ferroptosis mechanism (19).
Long non-coding RNAs (lncRNAs) are RNAs more than 200 nucleotides in length that regulate diverse physiological cellular processes through nucleotide mutation or gene expression alteration (20). Multiple studies have shown functions of lncRNAs in various human diseases, including cancers, through their ability to modulate key cellular processes such as ferroptosis. For instance, according to $\mathrm{Wu}$ et al., the lncRNA NEAT1 regulates ferroptosis sensitivity in lung cancer (21). Mao et al. demonstrated that the lncRNA P53RRA is down-regulated in cancers and functions as a tumor suppressor by promoting ferroptosis in cancers (22). Moreover, the lncRNA LINC00336 inhibits ferroptosis in lung cancer by acting as a competing endogenous RNA (23). Furthermore, research demonstrated that the lncRNAs GABPB1-AS1 and GABPB1 correlates with OS and regulates oxidative stress by ferroptosis in hepatocellular carcinoma (24). However, studies on the ferroptosis-related lncRNAs in OC patients have not been well explored.

The focus of this research was to explore ferroptosisrelated lncRNAs in OC and assess their prognostic significance. The Cancer Genome Atlas (TCGA) database was used to collect OC patient samples and RNA sequencing data. The patients were assigned to training and testing sets. In the training set, a predictive model using 11 ferroptosis-related lncRNAs was established, and the signature was subsequently validated using the testing and entire set. A nomogram combining the prognostic model and other clinical features was also evaluated. Functional enrichment analysis was carried out to explore underlying molecular mechanisms. We present the following article in accordance with the TRIPOD reporting checklist (available at https://dx.doi.org/10.21037/tcr-21-1152).

\section{Methods}

\section{Data collection}

The TCGA database (https://portal.gdc.cancer.gov/ repository) was used to obtain RNA sequencing data with clinical information of OC patients. Patients with a survival of less than 30 days were excluded (25). A total of 365 samples were included for subsequent analyses. The samples were randomly divided into the training set $(\mathrm{n}=184)$ and testing set $(\mathrm{n}=181)$. Annotation of lncRNAs was acquired from GENCODE (https://www.gencodegenes. org/). Ferroptosis-related genes were obtained from FerrDb, a web-based ferroptosis marker database that is comprehensive and up-to-date (http://www.zhounan.org/ 
ferrdb). The study was conducted in accordance with the Declaration of Helsinki (as revised in 2013).

\section{Identification of the ferroptosis-related lncRNA prognostic signature}

The association of lncRNAs with ferroptosis-related genes was evaluated by Pearson correlation analysis. A Pearson correlation coefficient (PCC) greater than 0.3 and a $\mathrm{P}$-value smaller than 0.001 were used to identify ferroptosis-related lncRNAs (26-28). Univariate Cox regression method was performed to analyze the prognostic role of the ferroptosisrelated lncRNAs. Least absolute shrinkage and selection operator (LASSO) Cox regression was then conducted for further screening from the results of the univariate Cox regression. To determine the final prognostic signature, multivariate Cox analysis with Akaike information criterion to avoid overfitting was performed. The risk score was calculated using the algorithm below: risk score $=\sum_{i=1}^{11}(X i * Y i)$ (X: coefficients; Y: expression level of lncRNA). OC patients were assigned to high-risk and low-risk groups according to the cut-off value (median risk score).

\section{Construction of the nomogram}

A prognostic nomogram combining the prognostic signature and several clinical indexes was established to forecast the OS of OC patients. Furthermore, the calibration plot and concordance index were applied to test the accuracy of the nomogram.

\section{PCA and GSEA}

Principal component analysis (PCA) was used to reduce dimension and visualize the different ferroptosis status of the high- and low-risk groups. Gene set enrichment analysis (GSEA) was conducted to investigate whether the genes were differentially expressed between the high- and low-risk groups.

\section{Statistical analysis}

Statistical analyses were performed using $\mathrm{R}$ version 4.0.2. Pearson correlation analysis was performed to evaluate the association of lncRNAs with ferroptosis-related genes. The OS of the high-risk and low-risk groups was compared by Kaplan-Meier survival analysis with a log-rank test. The sensitivity and specificity of the prognostic signature were evaluated using the receiver operating characteristics (ROC) curve and the area under the ROC (AUC). The association of the prognostic signature with other clinical features was also analyzed and the difference between the groups was assessed using Student's $t$-test. The independent prognostic significance of the prognostic signature was assessed though univariate and multivariate Cox regression models. $P<0.05$ was regarded as statistically significant.

\section{Results}

\section{Screening of ferroptosis-related lncRNAs in OC}

A total of 14,142 lncRNAs were extracted from TCGA database and 245 ferroptosis-related genes were collected from FerrDb. The association of IncRNAs with ferroptosisrelated genes was assessed through Pearson correlation analysis, and the analysis identified 1,249 ferroptosis-related lncRNAs.

\section{Development and evaluation of the prognostic signature in OC patients in the training set}

Univariate Cox regression analysis was applied to investigate 58 ferroptosis-related lncRNAs that had prognostic significance for OC in the training set (Figure 1A). LASSO Cox regression analysis was performed for further screening and identified 19 lncRNAs (Figure 1B,1C). Multivariate Cox regression analysis was then performed, resulting in a final 11 lncRNAs (Figure 1D).

We next examined the OS of patients according to expression of the lncRNAs, stratified by median expression. As shown in Table 1 and Figure 2, patients with high expression of AC007848.1, AC011445.1, and AC093895.1 had worse survival than those with low expression, and patients with high expression of AC010336.5, AL157871.2, AP001033.1, AC009403.1, AC068792.1, LINC01857, LINC00239, and AL513550.1 had better survival than patients with low expression.

The following ferroptosis-related lncRNA prognostic signature was established for OC: risk score $=(0.4555$ $\times$ AC007848.1 expression $)+(-0.2409 \times \mathrm{AC} 010336.5$ expression $)+(-0.6739 \times$ AL157871.2 expression $)+(-0.3055$ $\times$ AP001033.1 expression $)+(-0.1638 \times$ AC009403.1 expression $)+(-0.2275 \times$ AC068792.1 expression $)+(0.1263$ $\times$ AC011445.1 expression $)+(0.5263 \times$ AC093895.1 expression $)+(-0.1943 \times$ LINC01857 expression $)+(-0.2508$ 
A

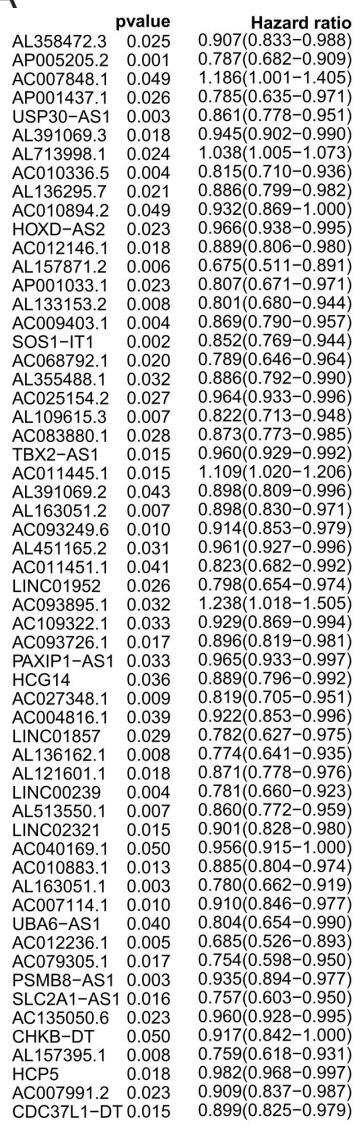

$1 \quad B$

B $\quad 5655524847423629251916146321$

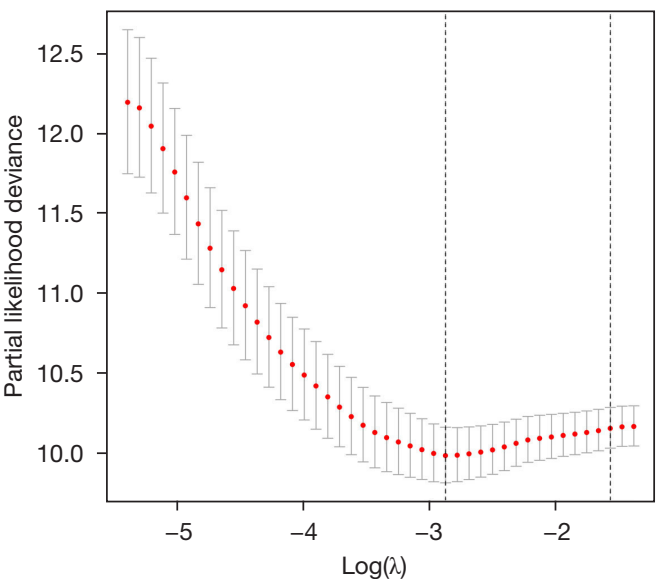

5655524847423629251916146321

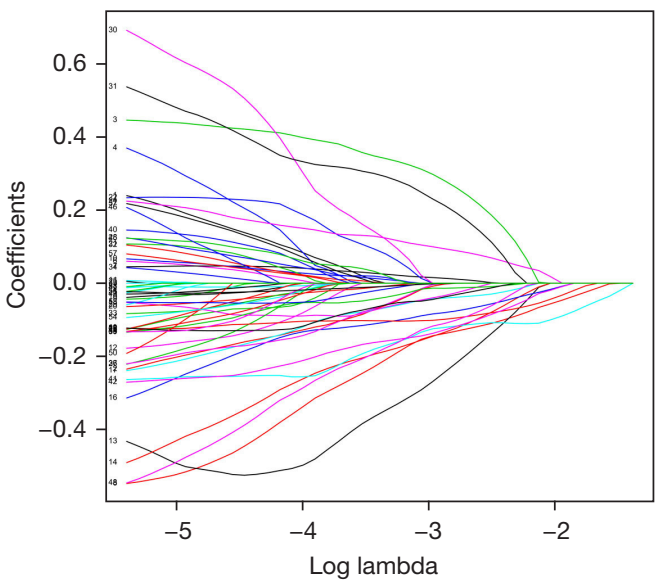

D

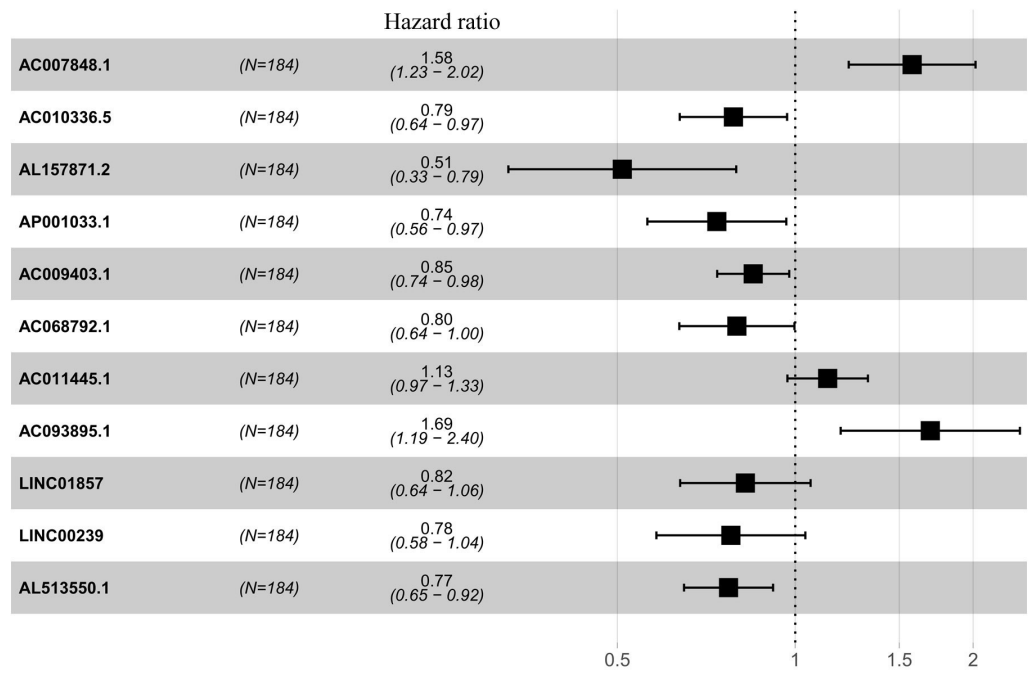

Figure 1 Identification of the ferroptosis-related lncRNA prognostic signature. (A) Univariate Cox regression analysis of OS for 58 ferroptosis-related lncRNAs. Red bar represents the size of HR value (HR $>1)$, and green bar represents the size of HR value $(H R<1)$. (B, C) Altogether 19 lncRNAs were screened using LASSO regression. (D) Multivariate Cox regression forest plot of the 11 ferroptosis-related lncRNAs. OS, overall survival; HR, hazard ratio; LASSO, least absolute shrinkage and selection operator. 
Table 1 Multivariate Cox results of the 11 ferroptosis-related lncRNAs

\begin{tabular}{lccc}
\hline LncRNA & coef & HR & $95 \% \mathrm{Cl}$ \\
\hline AC007848.1 & 0.4555 & 1.5770 & $1.2318-2.0191$ \\
AC010336.5 & -0.2409 & 0.7859 & $0.6376-0.9688$ \\
AL157871.2 & -0.6739 & 0.5097 & $0.3270-0.7945$ \\
AP001033.1 & -0.3055 & 0.7367 & $0.5620-0.9658$ \\
AC009403.1 & -0.1638 & 0.8486 & $0.7377-0.9769$ \\
AC068792.1 & -0.2275 & 0.7966 & $0.6365-0.9969$ \\
AC011445.1 & 0.1263 & 1.1347 & $0.9699-1.3274$ \\
AC093895.1 & 0.5263 & 1.6926 & $1.1937-2.3999$ \\
LINC01857 & -0.1943 & 0.8234 & $0.6386-1.0617$ \\
LINC00239 & -0.2508 & 0.7782 & $0.5821-1.0402$ \\
AL513550.1 & -0.2599 & 0.7711 & $0.6483-0.9172$ \\
\hline
\end{tabular}

$\times$ LINC00239 expression $)+(-0.2599 \times$ AL5 13550.1 expression). The prognostic risk score for each patient was calculated using the formula described above in the training set, and the median score was utilized as the threshold to divide the patients into high- and low-risk groups. The distribution patterns of risk scores and survival of the 184 OC patients in the training set are shown in Figure $3 A, 3 B$, and the expression of the 11 lncRNAs in the high- and lowrisk groups is shown in a heatmap in Figure $3 C$. Patients in the high-risk group had a poorer OS rate than those in the low-risk group according to the Kaplan-Meier analysis (Figure 3D). The accuracy of the risk score model was assessed using ROC curve analysis, with AUC values of $0.731,0.796$, and 0.805 for 1-, 3-, and 5-year OS rates, respectively (Figure 3E).

\section{Validation of the prognostic signature in the testing set and the entive set}

The predictive ability of the prognostic signature was assessed in both the testing set $(n=181)$ and the entire set $(n=365)$. The risk scores of patients in the testing set and entire set were calculated in the same way as in the training set, and patients were also assigned to high- and low-risk subgroups based on the same cutoff value in the training set. In the testing set, Figure $4 A-4 C$ showed the distributions of the risk scores, survival status and the expression heatmap for the 11 lncRNAs, and Figure $4 D$ exhibited a substantial difference in OS between the high- and low-risk groups. In addition, the AUCs for 1-, 3-, and 5 -year OS rates were $0.704,0.597$, and 0.655 , respectively (Figure $4 E$ ). These findings indicated that the predictive signature was accurate and robust. Similarly, results from the entire set also demonstrated the robustness of this prognostic model (Figure 5A-5D) and Figure 5E also showed the accuracy of the signature by the AUCs $(0.715$ for 1-year OS, 0.691 for 3-year OS, and 0.736 for 5 -year OS, respectively).

\section{Association of the prognostic signature with clinical features and independence of the prognostic signature}

We focused on the exploration of the association of ferroptosis-related lncRNA prognostic signature with age and pathological grade in OC patients. The risk score was significantly higher in the age $\geq 65$ years group than the age $<65$ years group $(\mathrm{P}=0.047$, Figure $6 A)$ and higher in the grade III-IV group than the grade I-II group $(\mathrm{P}=0.045$, Figure $6 B)$. The independent value of the prognostic signature was assessed by univariate and multivariate Cox regression analyses. Univariate regression analysis showed that age (HR: $1.021,95 \%$ CI: $1.008-1.034, \mathrm{P}=0.002$ ) and risk score (HR: 2.026, 95\% CI: 1.708-2.404, $\mathrm{P}<0.001)$ were associated with OS of OC patients (Figure 6C). Multivariate Cox regression analysis showed that age (HR: $1.015,95 \%$ CI: $1.002-1.028, \mathrm{P}=0.021)$ and risk score (HR: $1.940,95 \%$ CI: $1.635-2.302, \mathrm{P}<0.001)$ were independent prognostic factors for OC patients (Figure 6D).

\section{Nomogram}

A nomogram combining age, pathological grade, and risk score to predict 1-, 3-, and 5-year OS for OC patients was generated (Figure 7A). The concordance index of the prognostic model was 0.68 . The calibration curve showed that the observed and the predicted OS were consistent (Figure $7 B-7 D$ ).

\section{PCA and GSEA}

PCA was performed to analyze the scatter patterns between low- and high-risk groups based on the entire genome expression set, ferroptosis-related gene set, ferroptosisrelated lncRNA set, and ferroptosis-related prognostic lncRNA set (Figure 8). The results showed that the OC patients were assigned into two clusters based on the model lncRNAs. 

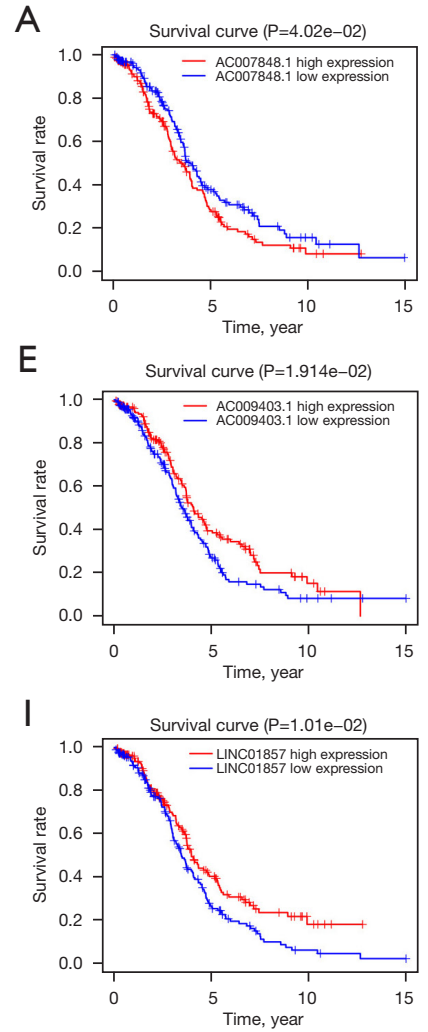

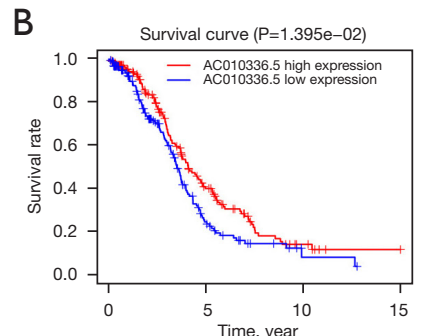

$\mathrm{F}$
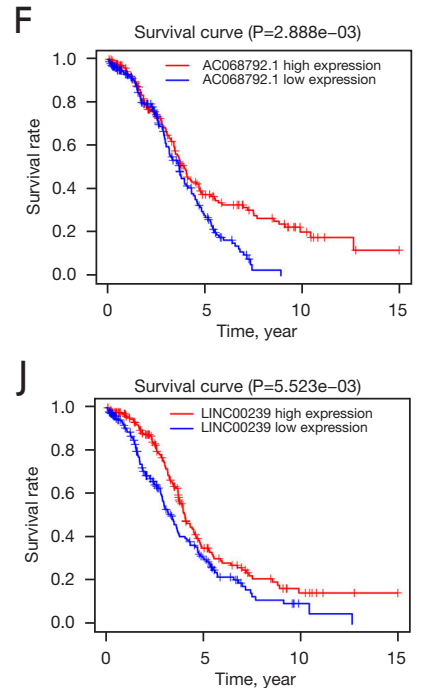

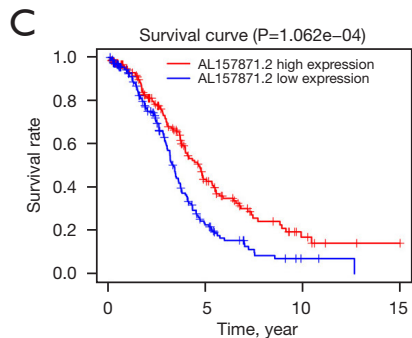

G
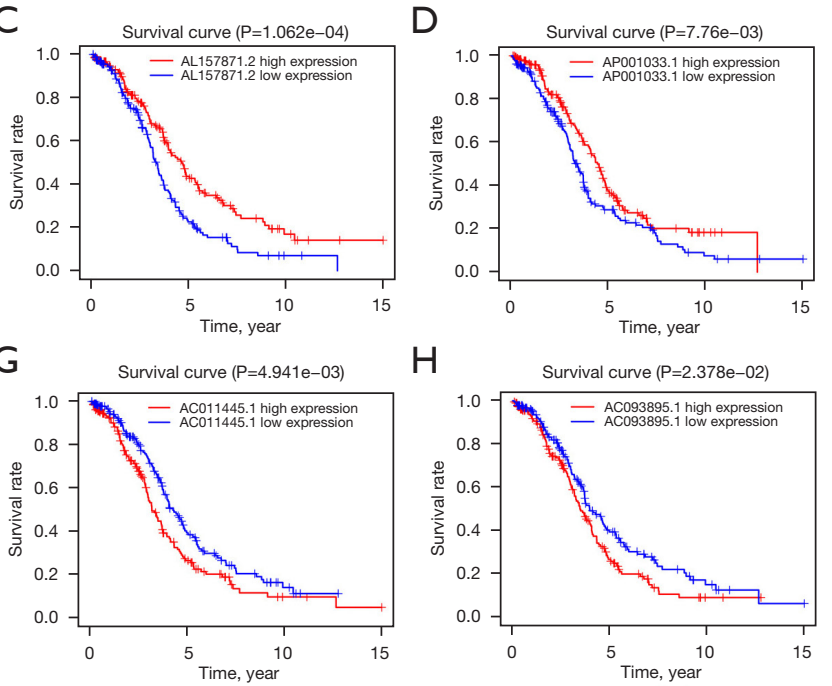

$\mathrm{H}$
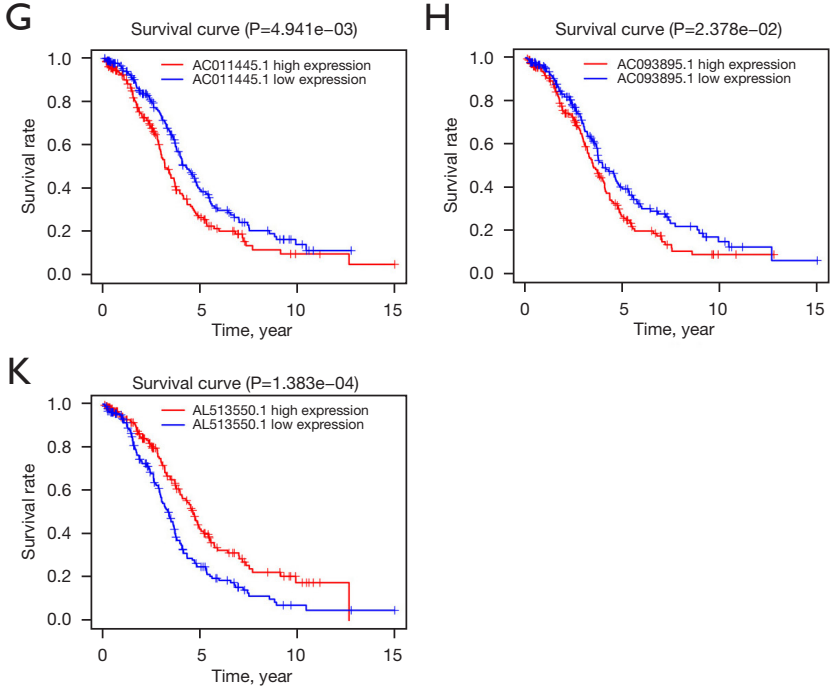

Figure 2 Kaplan-Meier survival analysis of OC patients according to expression of the 11 ferroptosis-related lncRNAs. (A) AC007848.1; (B) AC010336.5; (C) AL157871.2; (D) AP001033.1; (E) AC009403.1; (F) AC068792.1; (G) AC011445.1; (H) AC093895.1; (I) LINC01857; (J) LINC00239; (K) AL513550.1. OC, ovarian cancer.

To study the physiological mechanism and pathways of the differentially expressed genes in the prognostic signature, we conducted GSEA. The results showed that axon guidance, colorectal cancer, endometrial cancer, long term potentiation, and thyroid cancer signal paths were centralized in the high-risk group, while primary immunodeficiency and protein export signal paths were centralized in the low-risk group (Figure 9).

\section{Discussion}

$\mathrm{OC}$ is the third most occurring cancer in the female genital system after cervical cancer and endometrial cancer and has the highest rate of cancer-related deaths in women compared with other gynecological cancers (29). In recent years, lncRNAs have been shown to act as biomarkers for the diagnosis and prognosis in OC. For example, the lncRNA TOPORS-AS1, which suppresses the $\mathrm{Wnt} / \beta$-catenin axis, is linked to a better prognosis in OC patients (30). According to $\mathrm{Li}$ et al., upregulation of 1ncRNA DUXAP8 promotes the proliferation and migration by down-regulating microRNA-29a-3p and correlated with a poor prognosis in OC patients (31). Jiang et al. demonstrated the carcinogenic role of the lncRNA XIST in OC and showed that high expression of XIST indicates a poor prognosis for OC patients (32). Moreover, studies showed that dysregulation of LINC00152 is a risk factor for poor outcome in patients with OC (33) and the lncRNA E2F4as predicts poor prognosis and promotes OC progression (34). These results demonstrate that lncRNAs are promising prognostic markers in OC. An autophagy-related lncRNA prognostic signature for OC was constructed by Meng et al. (35) and an immune-related lncRNA prognostic model for the prediction of survival in OC was established by Liang et al. (36). However, it appears there is no evidence of a ferroptosis-related lncRNA prognostic signature in OC.

In this study, we used the TCGA database to collect 
A

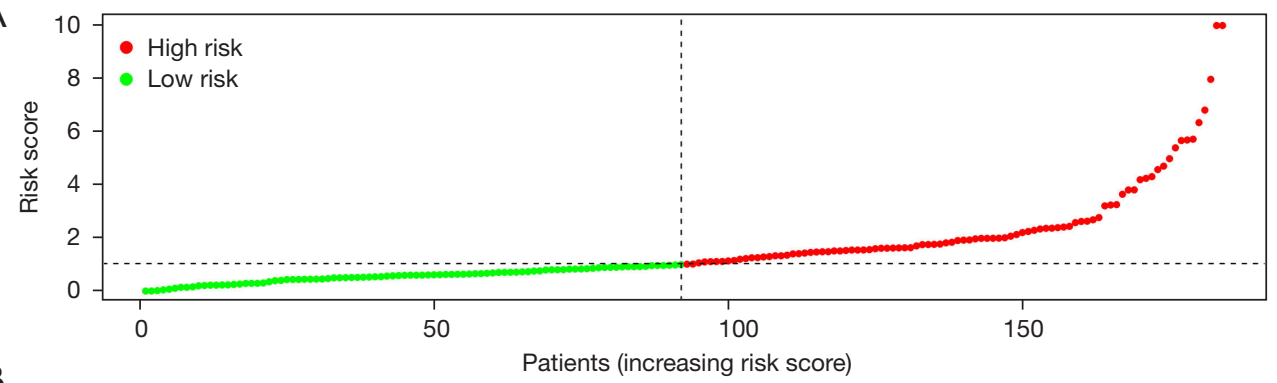

B

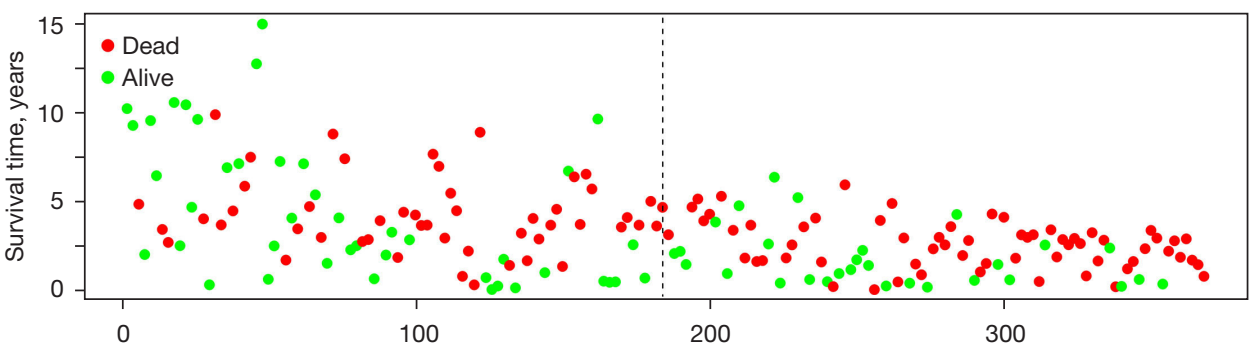

C

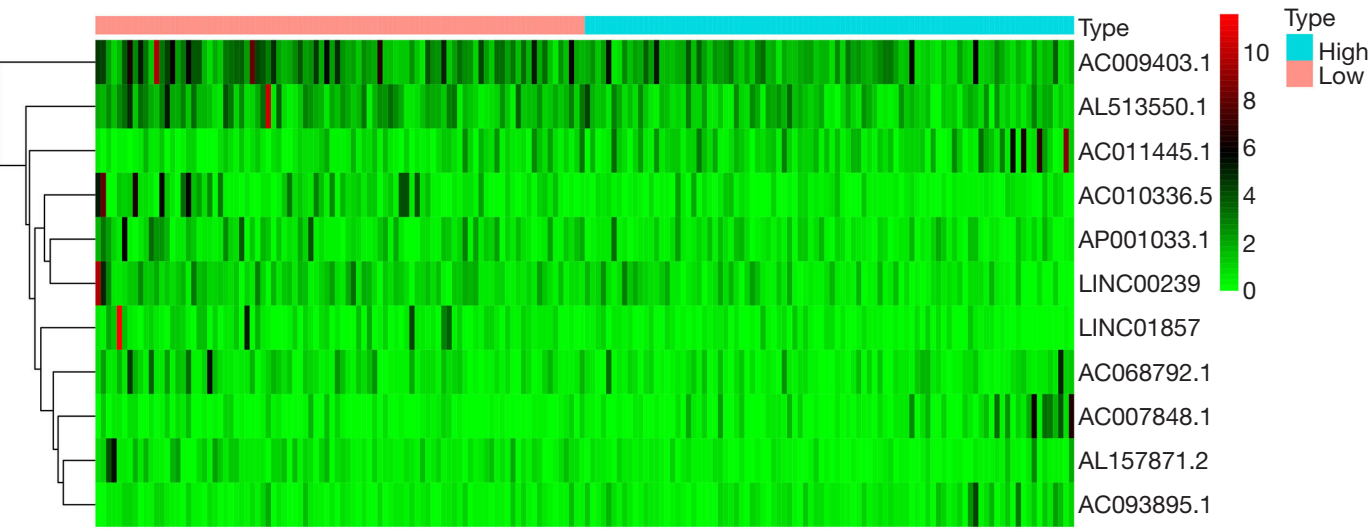

D

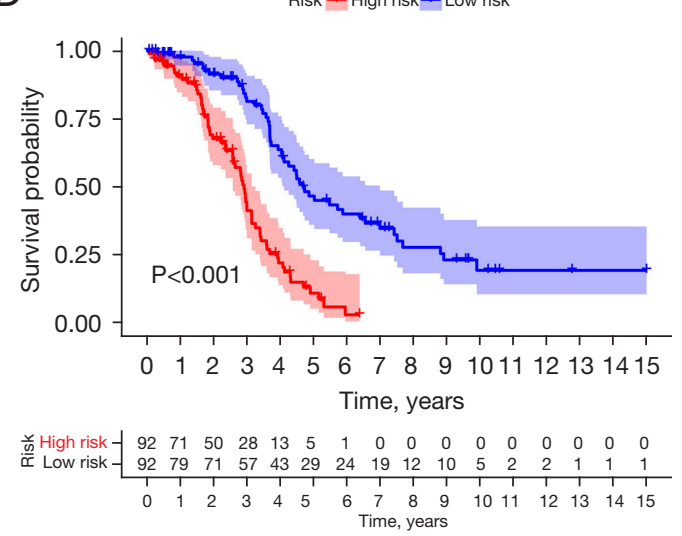

E

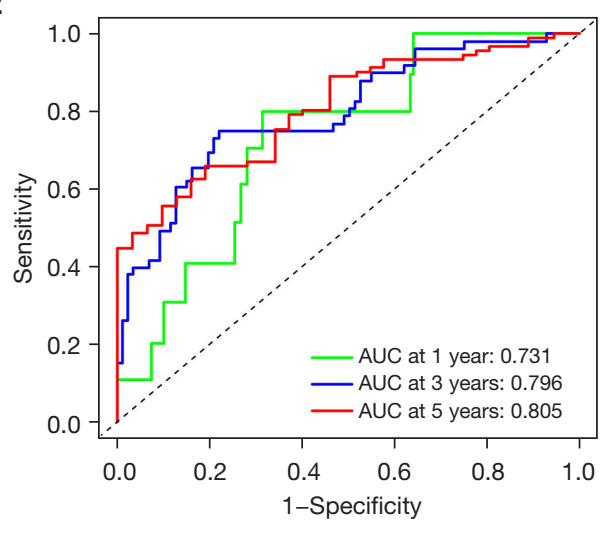

Figure 3 Assessment of the prognostic signature in the training set. (A) The OC patients' risk score distribution. (B) Survival status of the OC patients. (C) Heatmap to show the expression of 11 lncRNAs between high- and low-risk groups. (D) A Kaplan-Meier survival analysis for evaluating the OS of patients in low- and high-risk groups. (E) ROC curve analysis for OC patients. OC, ovarian cancer; OS, overall survival; ROC, receiver operating characteristics. 
A

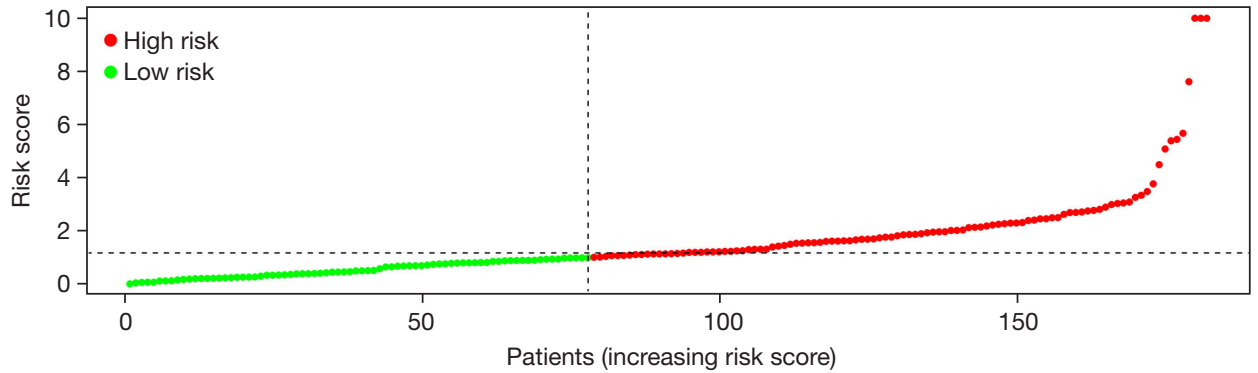

B

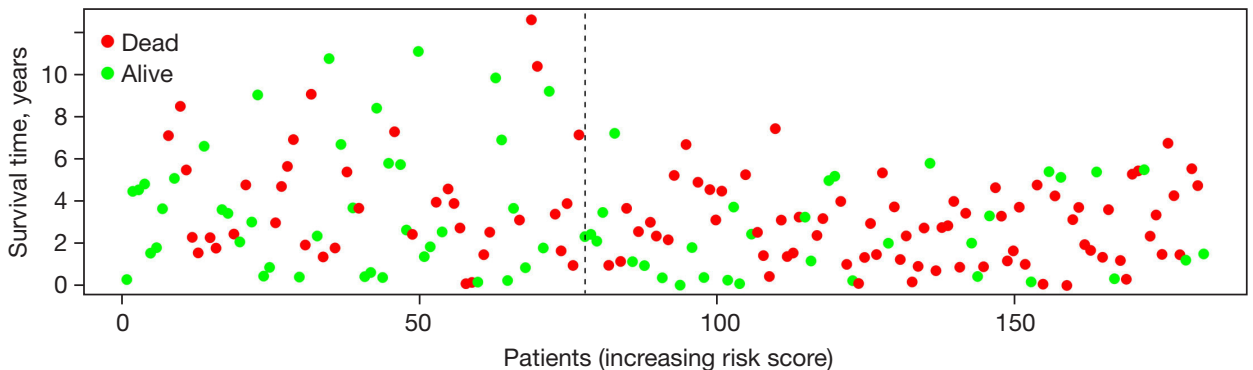

C

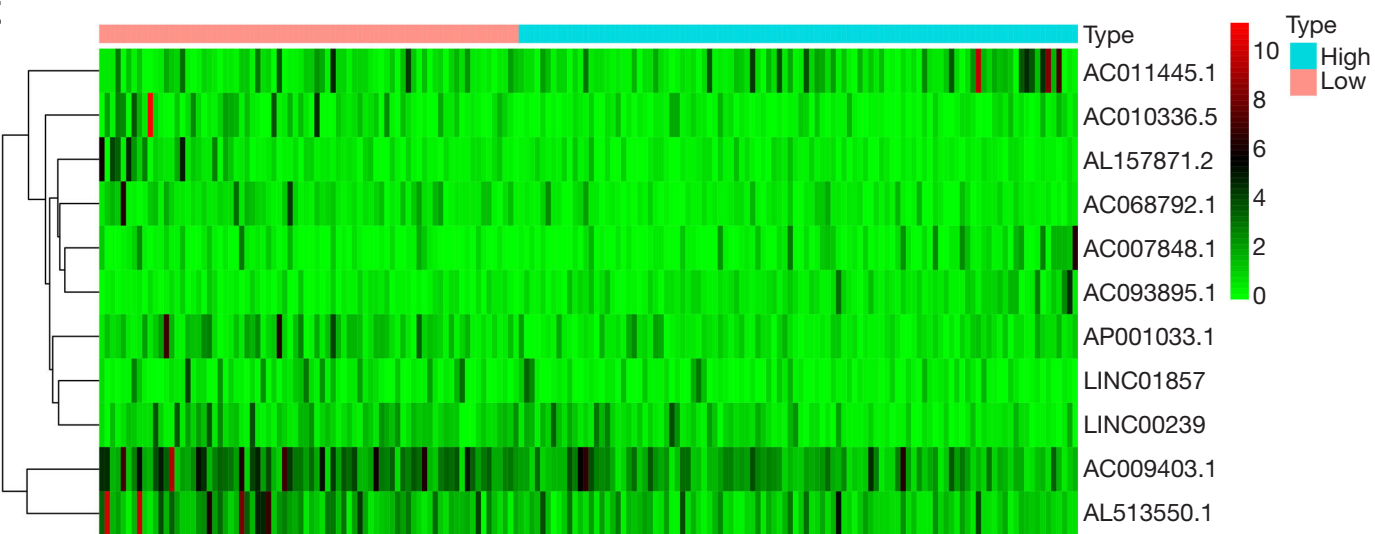

D

Risk $=$ High risk - Low risk

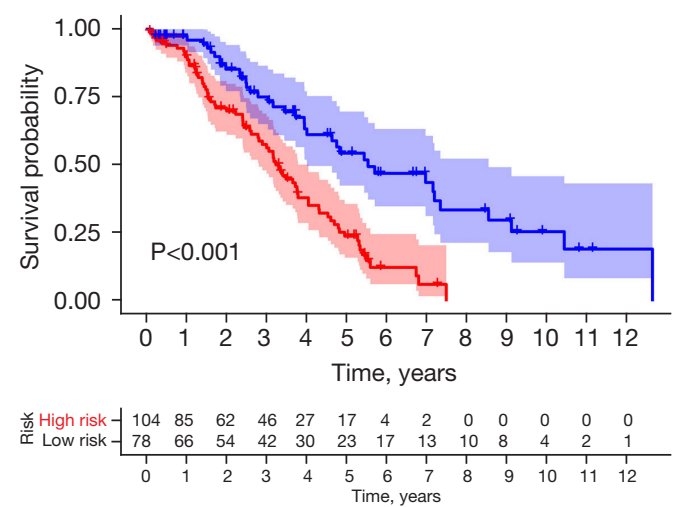

E

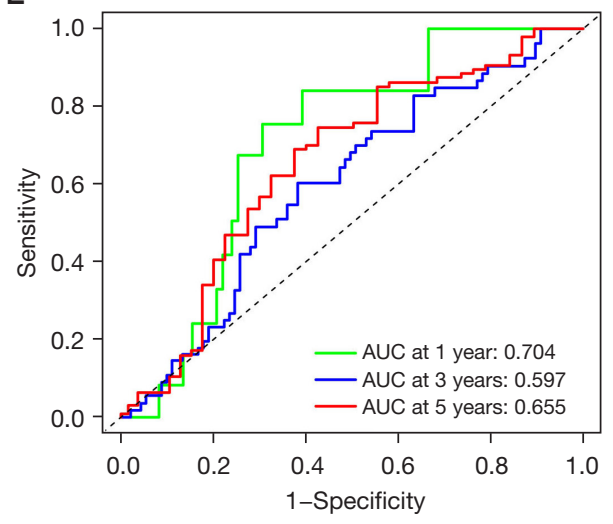

Figure 4 Validation of the ferroptosis-related lncRNA signature in the testing set. (A) The OC patients' risk score distribution. (B) Survival status of the OC patients. (C) Heatmap to show the expression of 11 lncRNAs between high- and low-risk groups. (D) A Kaplan-Meier survival analysis for evaluating the OS of patients in low- and high-risk groups. (E) ROC curve analysis for OC patients. OC, ovarian cancer; OS, overall survival; ROC, receiver operating characteristics. 
A

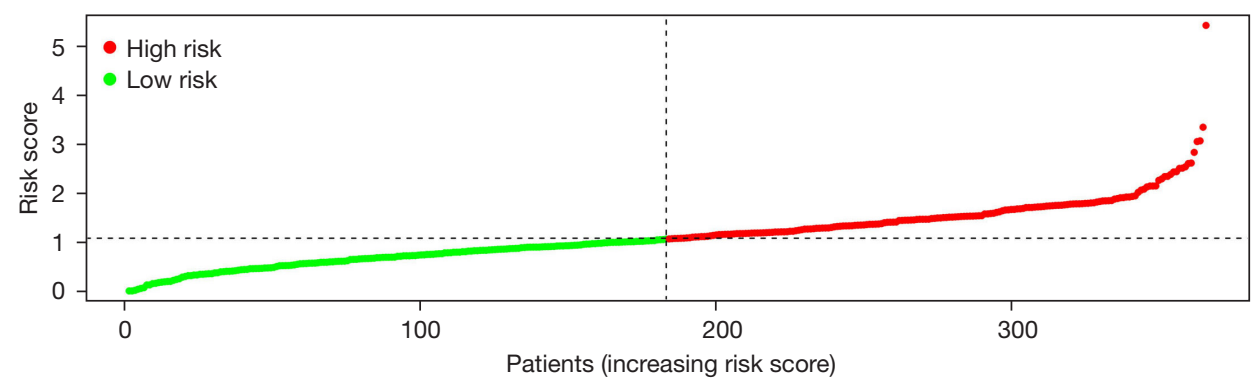

B

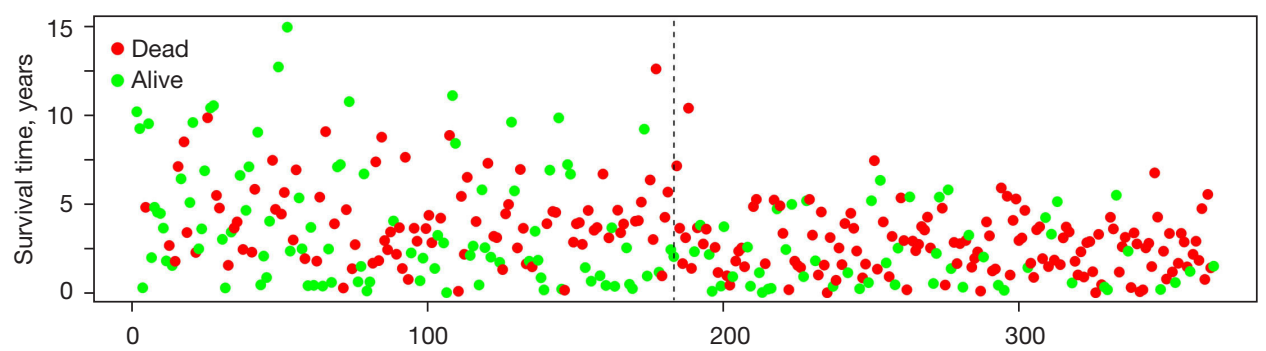

C

Patients (increasing risk score)

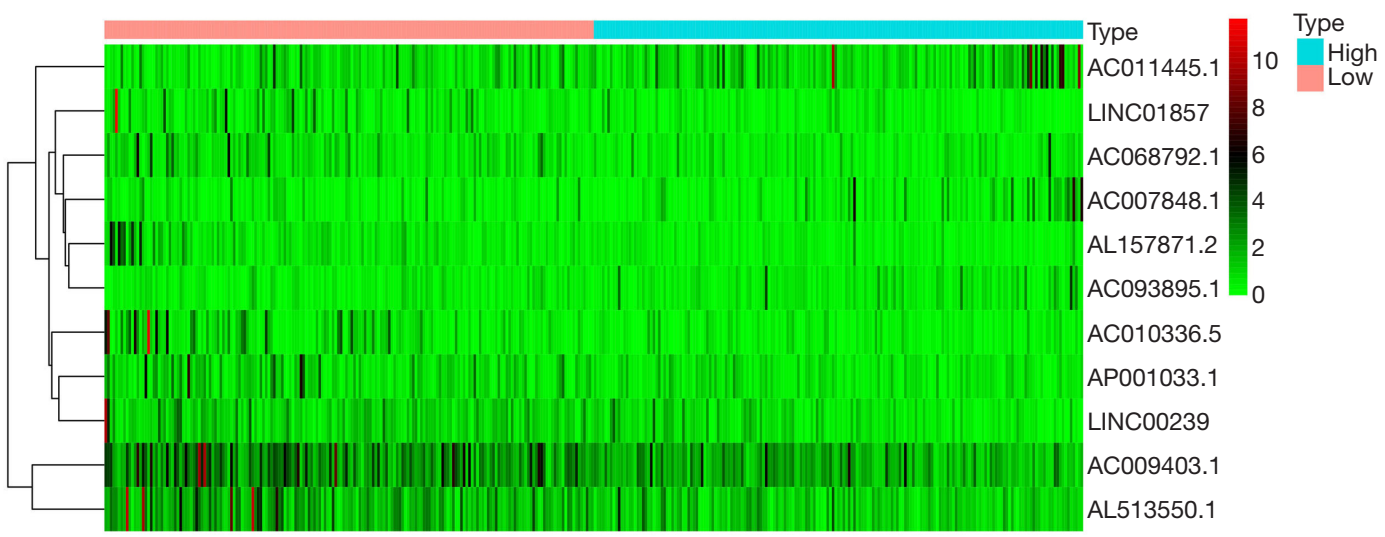

D

Risk + High risk - Low risk

$\mathrm{E}$

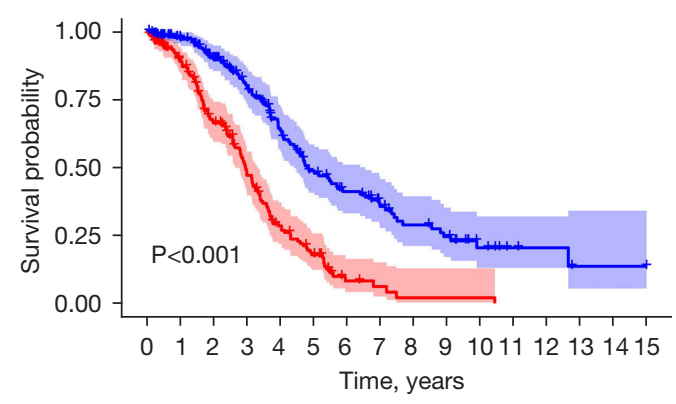

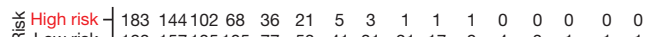

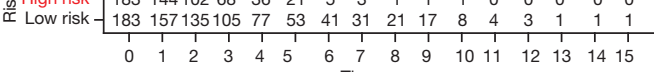

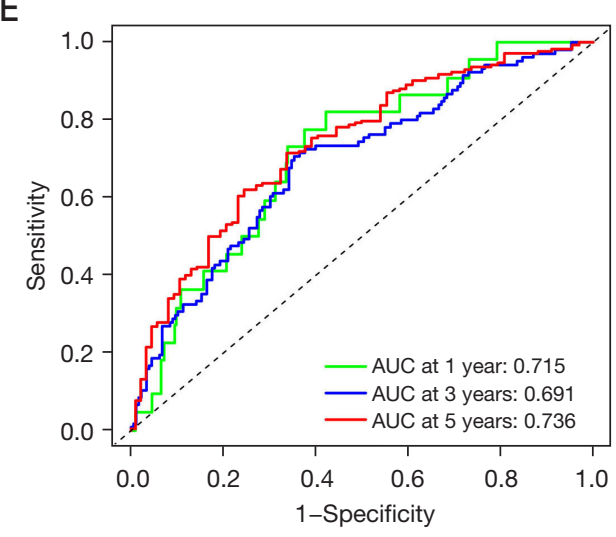

Figure 5 Validation of the ferroptosis-related lncRNA signature in the entire set. (A) The OC patients' risk score distribution. (B) Survival status of the OC patients. (C) Heatmap to show the expression of 11 lncRNAs between high- and low-risk groups. (D) A Kaplan-Meier survival analysis for evaluating the OS of patients in low- and high-risk groups. (E) ROC curve analysis for OC patients. OC, ovarian cancer; OS, overall survival; ROC, receiver operating characteristics. 
A
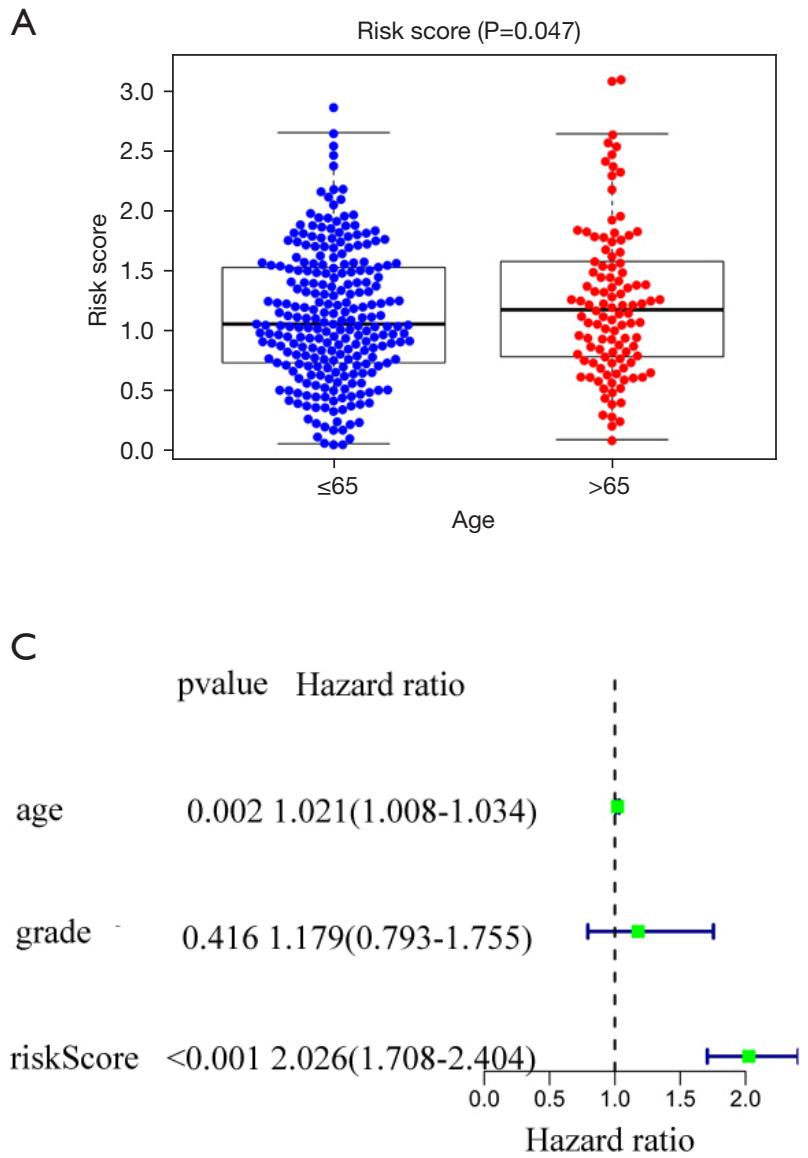

B

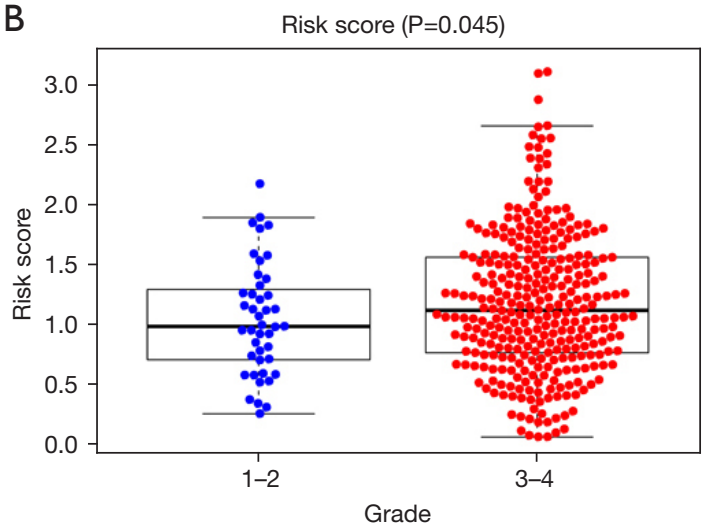

age

$0.0211 .015(1.002-1.028)$

grade

$0.5181 .139(0.767-1.692)$

pvalue Hazard ratio

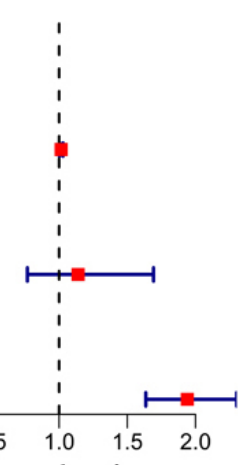

riskScore

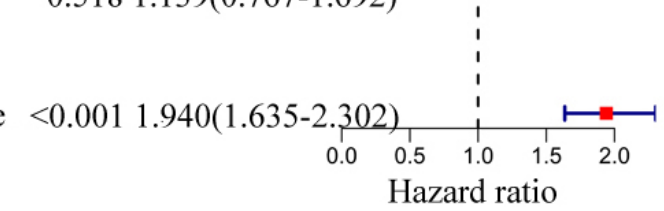

Figure 6 Association of the prognostic signature with clinical features. (A) With age; (B) with pathological grade; (C) forest plots of univariate Cox analysis; (D) forest plots of multivariate Cox analysis.

IncRNA expression data of OC patients and the FerrDb dataset to acquire ferroptosis-related genes. We assessed the association of IncRNAs with ferroptosis-related genes by carrying out Pearson correlation analysis for screening of ferroptosis-related lncRNAs. OC patient samples were randomly allocated into training and testing sets. In the training set, a ferroptosis-related lncRNA predictive signature was established by univariate Cox, LASSO, and multivariate Cox regression. Patients were assigned into high- and low-risk groups based on the median risk scores, and those in the high-risk group had a shorter survival time than those in the low-risk group. Furthermore, a good AUC value suggested that the joint predictive ability of this 11 ferroptosis-related lncRNAs prognostic model for OC was highly reliable. The prognostic model was also verified in the testing set and the entire set with survival analysis and suitable AUC values. Therefore, this prognostic signature was a robust model in predicting prognosis for patients with OC. Moreover, the results showed that the ferroptosisrelated lncRNA prognostic signature was an independent prognostic factor and correlated with clinical features.

A total of 11 lncRNAs including AC007848.1, AC011445.1, AC093895.1, AC010336.5, AL157871.2, AP001033.1, AC009403.1, AC068792.1, LINC01857, LINC00239, and AL513550.1 were identified for the prognostic signature. Studies reported that LINC01857 promotes cell growth and tumor development in diffuse large B-cell lymphoma (37), gastric cancer (38), glioma (39), and breast cancer (40). LINC00239 was an oncogene in myeloid leukemia (41) and colorectal cancer (42). However, the role of LINC01857 and LINC00239 in OC has not been previously reported. The association of the other 9 lncRNAs with OC was first demonstrated in this study. The GSEA results demonstrated that cancer-related pathways 
A

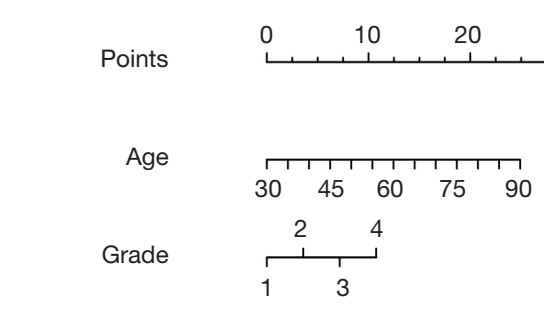

Risk score

\begin{tabular}{llllllllllll}
\hline 0 & 0.5 & 1 & 1.5 & 2 & 2.5 & 3 & 3.5 & 4 & 4.5 & 5 & 5.5
\end{tabular}

Total points

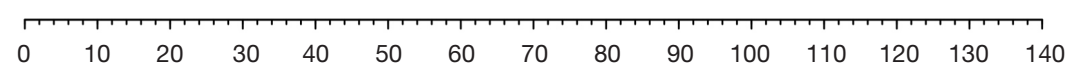

1-year survival

3-year survival

\begin{tabular}{lllll}
\hline & 1 & 1 & \\
0.9 & 0.8 & 0.7 & 0.5 & 0.3
\end{tabular}

5-year survival
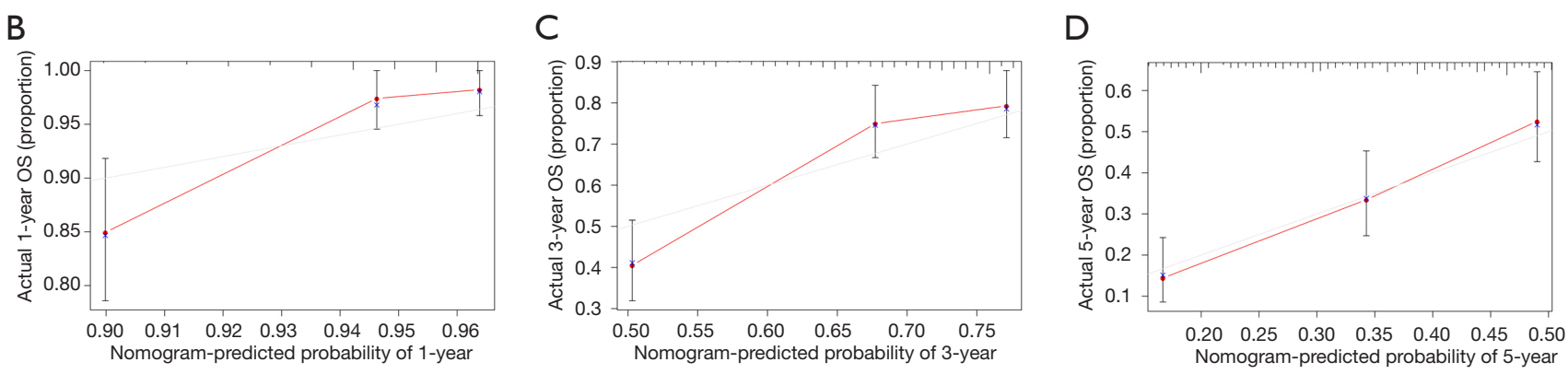

Figure 7 Nomogram development and evaluation. (A) A nomogram for predicting OS in OC patients based on age, pathological grade, and risk score. The calibration curve for determining the strength of the nomogram's power to predict 1-year (B), 3-year (C), and 5-year (D) OS. OS, overall survival; OC, ovarian cancer.

including colorectal cancer, endometrial cancer, and thyroid cancer pathways were significantly enriched in the high-risk group. Moreover, axon guidance and long-term potentiation pathways, which are considered to be related to tumor development (43-45), were also enriched in the high-risk group. These findings suggested that the 11 ferroptosisrelated lncRNAs are associated with the occurrence and development of OC.

The present study had some limitations. The results obtained in this study were solely based on a TCGA dataset with a relatively small sample size. It is ideal to validate this prognostic model through an independent dataset. Unfortunately, the other independent dataset of lncRNAs with prognostic information on OC could not be found. Only the TCGA testing set and the entire set were used to test the availability of this IncRNA prognostic signature. Therefore, other prospective clinical cohorts need to be conducted to confirm the robustness of this prognostic model in the future. In addition, ferroptosis is regulated by many gene products including coding and non-coding transcripts and how much this signature accounts for the process of ferroptosis compared to the involvement of mRNAs or the combination of mRNAs and lncRNAs is still unknown. Thus, experimental studies will also be required to explore the molecular mechanism of the ferroptosisrelated lncRNAs in OC.

In summary, we identified 11 ferroptosis-related lncRNAs in OC and developed a signature based on the 11 lncRNAs that had independent prognostic value for OC patients. 
A

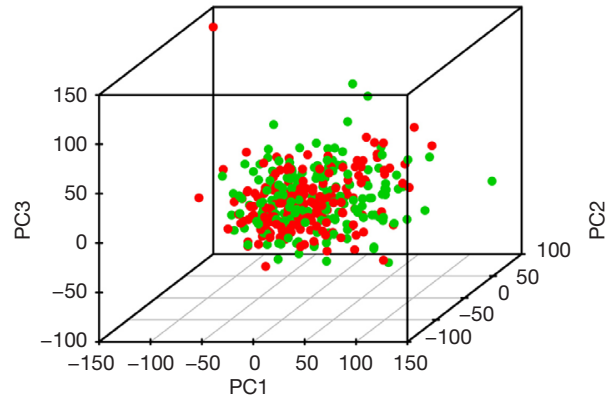

C

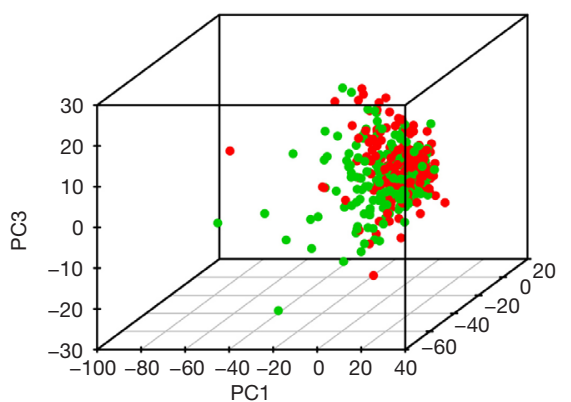

B

- Low risk • High risk

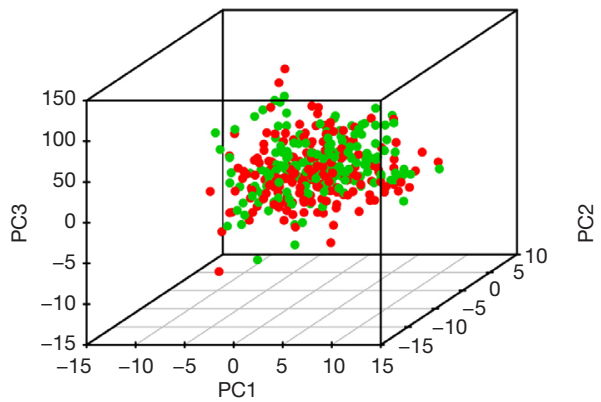

$\mathrm{D}$

- Low risk - High risk

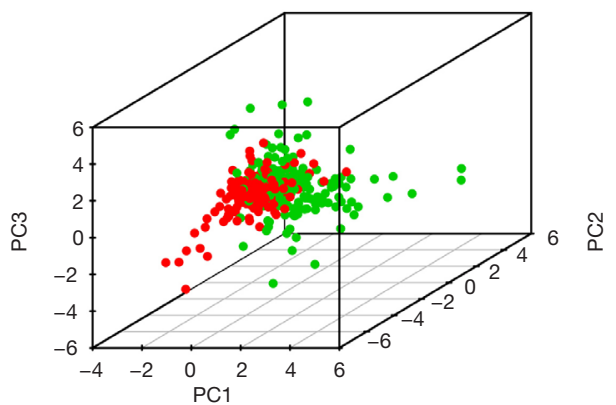

Figure 8 PCA plot for the distribution patterns between low- and high-risk groups based on the whole genome expression set (A), ferroptosis-related gene set (B), ferroptosis-related lncRNA set (C), and ferroptosis-related prognostic lncRNA set (D). PCA, principal component analysis.

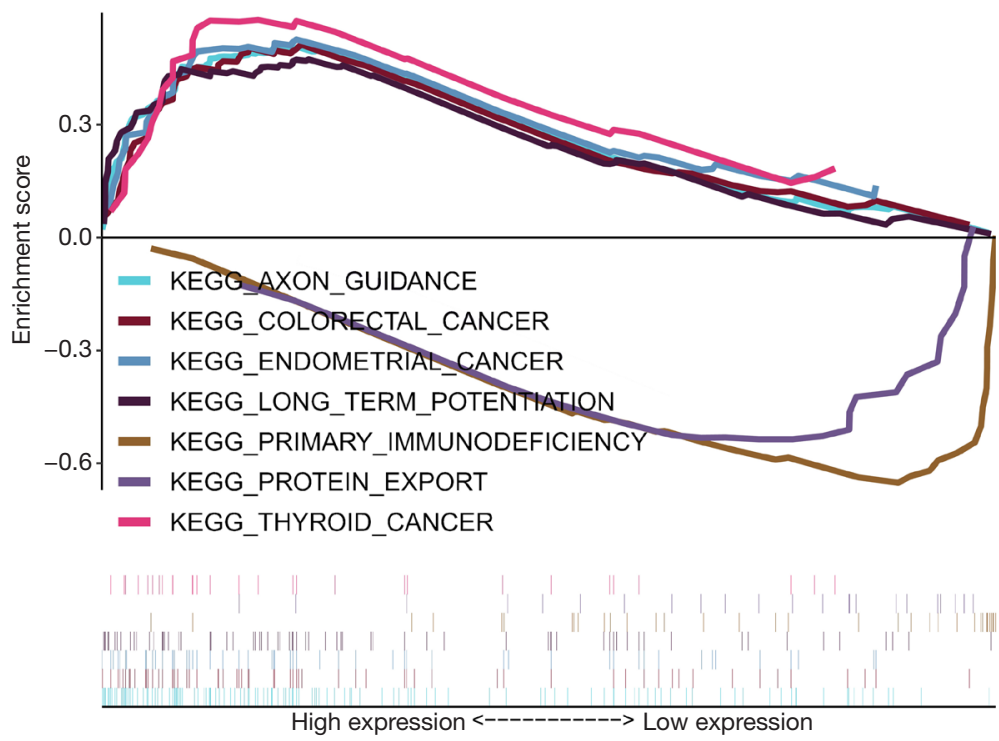

Figure 9 GSEA showed the pathways that are enriched in the high-risk group and low-risk group. GSEA, gene set enrichment analysis. 


\section{Acknowledgments}

We thank Gabrielle White Wolf, $\mathrm{PhD}$, from Liwen Bianji, Edanz Editing China (www.liwenbianji.cn/ac), for editing the English text of a draft of this manuscript.

Funding: This work was supported by the National Natural Science Foundation of China (82071724).

\section{Footnote}

Reporting Checklist: The authors have completed the TRIPOD reporting checklist. Available at https://dx.doi. org/10.21037/tcr-21-1152

Conflicts of Interest: All authors have completed the ICMJE uniform disclosure form (available at https://dx.doi. org/10.21037/tcr-21-1152). The authors have no conflicts of interest to declare.

Ethical Statement: The authors are accountable for all aspects of the work in ensuring that questions related to the accuracy or integrity of any part of the work are appropriately investigated and resolved. The study was conducted in accordance with the Declaration of Helsinki (as revised in 2013).

Open Access Statement: This is an Open Access article distributed in accordance with the Creative Commons Attribution-NonCommercial-NoDerivs 4.0 International License (CC BY-NC-ND 4.0), which permits the noncommercial replication and distribution of the article with the strict proviso that no changes or edits are made and the original work is properly cited (including links to both the formal publication through the relevant DOI and the license). See: https://creativecommons.org/licenses/by-nc-nd/4.0/.

\section{References}

1. Ye Y, Dai Q, Qi H. A novel defined pyroptosis-related gene signature for predicting the prognosis of ovarian cancer. Cell Death Discov 2021;7:71.

2. Sung H, Ferlay J, Siegel RL, et al. Global Cancer Statistics 2020: GLOBOCAN Estimates of Incidence and Mortality Worldwide for 36 Cancers in 185 Countries. CA Cancer J Clin 2021;71:209-49.

3. Lheureux S, Gourley C, Vergote I, et al. Epithelial ovarian cancer. Lancet 2019;393:1240-53.

4. Kuroki L, Guntupalli SR. Treatment of epithelial ovarian cancer. BMJ 2020;371:m3773.

5. Wei W, Li N, Sun Y, et al. Clinical outcome and prognostic factors of patients with early-stage epithelial ovarian cancer. Oncotarget 2017;8:23862-70.

6. Gao M, Monian P, Jiang X. Metabolism and iron signaling in ferroptotic cell death. Oncotarget 2015;6:35145-6.

7. Bednarska-Szczepaniak K, Przelazły E, Kania KD, et al. Interaction of Adenosine, Modified Using Carborane Clusters, with Ovarian Cancer Cells: A New Anticancer Approach against Chemoresistance. Cancers (Basel) 2021;13:3855.

8. Park JH, Kundu A, Lee SH, et al. Specific Pyruvate Kinase M2 Inhibitor, Compound 3K, Induces Autophagic Cell Death through Disruption of the Glycolysis Pathway in Ovarian Cancer Cells. Int J Biol Sci 2021;17:1895-908.

9. Yin L, Wang Y. Long non-coding RNA NEAT1 facilitates the growth, migration, and invasion of ovarian cancer cells via the let-7 g/MEST/ATGL axis. Cancer Cell Int 2021;21:437.

10. Stockwell BR, Jiang X, Gu W. Emerging Mechanisms and Disease Relevance of Ferroptosis. Trends Cell Biol 2020;30:478-90.

11. Dixon SJ, Lemberg KM, Lamprecht MR, et al. Ferroptosis: an iron-dependent form of nonapoptotic cell death. Cell 2012;149:1060-72.

12. Stockwell BR, Friedmann Angeli JP, Bayir H, et al. Ferroptosis: A Regulated Cell Death Nexus Linking Metabolism, Redox Biology, and Disease. Cell 2017;171:273-85.

13. Toyokuni S, Ito F, Yamashita K, et al. Iron and thiol redox signaling in cancer: An exquisite balance to escape ferroptosis. Free Radic Biol Med 2017;108:610-26.

14. Cramer SL, Saha A, Liu J, et al. Systemic depletion of L-cyst(e)ine with cyst(e)inase increases reactive oxygen species and suppresses tumor growth. Nat Med 2017;23:120-7.

15. Hassannia B, Vandenabeele P, Vanden Berghe T. Targeting Ferroptosis to Iron Out Cancer. Cancer Cell 2019;35:830-49.

16. Liang $C$, Zhang $X$, Yang $M$, et al. Recent Progress in Ferroptosis Inducers for Cancer Therapy. Adv Mater 2019;31:e1904197.

17. Wang Y, Zhao G, Condello S, et al. Frizzled-7 Identifies Platinum-Tolerant Ovarian Cancer Cells Susceptible to Ferroptosis. Cancer Res 2021;81:384-99.

18. Zhang Y, Xia M, Zhou Z, et al. p53 Promoted Ferroptosis in Ovarian Cancer Cells Treated with Human Serum Incubated-Superparamagnetic Iron Oxides. Int J 
Nanomedicine 2021;16:283-96.

19. Cheng Q, Bao L, Li M, et al. Erastin synergizes with cisplatin via ferroptosis to inhibit ovarian cancer growth in vitro and in vivo. J Obstet Gynaecol Res 2021;47:2481-91.

20. Shen XQ, Wu QM, Yang CH, et al. Four Low Expression LncRNAs are Associated with Prognosis of Human Lung Adenocarcinoma. Clin Lab 2020. doi: 10.7754/Clin. Lab.2020.200211.

21. Wu H, Liu A. Long non-coding RNA NEAT1 regulates ferroptosis sensitivity in non-small-cell lung cancer. J Int Med Res 2021;49:300060521996183.

22. Mao C, Wang X, Liu Y, et al. A G3BP1-Interacting lncRNA Promotes Ferroptosis and Apoptosis in Cancer via Nuclear Sequestration of p53. Cancer Res 2018;78:3484-96.

23. Wang M, Mao C, Ouyang L, et al. Long noncoding RNA LINC00336 inhibits ferroptosis in lung cancer by functioning as a competing endogenous RNA. Cell Death Differ 2019;26:2329-43.

24. Qi W, Li Z, Xia L, et al. LncRNA GABPB1-AS1 and GABPB1 regulate oxidative stress during erastin-induced ferroptosis in HepG2 hepatocellular carcinoma cells. Sci Rep 2019;9:16185.

25. You J, Fang W, Zhao Q, et al. Identification of a RNA-Seq Based Prognostic Signature with Seven Immune-Related lncRNAs for Lung Adenocarcinoma. Clin Lab 2021. doi: 10.7754/Clin.Lab.2020.200663.

26. Tang Y, Li C, Zhang YJ, et al. Ferroptosis-Related Long Non-Coding RNA signature predicts the prognosis of Head and neck squamous cell carcinoma. Int J Biol Sci 2021;17:702-11.

27. Wang X, Dai C, Ye M, et al. Prognostic value of an autophagy-related long-noncoding-RNA signature for endometrial cancer. Aging (Albany NY) 2021;13:5104-19.

28. Yao J, Chen X, Liu X, et al. Characterization of a ferroptosis and iron-metabolism related lncRNA signature in lung adenocarcinoma. Cancer Cell Int 2021;21:340.

29. Shen $W$, Xie X, Liu M, et al. Diagnostic Value of lncRNA ROR in Differentiating Ovarian Cancer Patients. Clin Lab 2020. doi: 10.7754/Clin.Lab.2019.191035.

30. Fu Y, Katsaros D, Biglia N, et al. Vitamin D receptor upregulates lncRNA TOPORS-AS1 which inhibits the Wnt/ $\beta$-catenin pathway and associates with favorable prognosis of ovarian cancer. Sci Rep 2021;11:7484.

31. Li LM, Hao SJ, Ni M, et al. DUXAP8 promotes the proliferation and migration of ovarian cancer cells via down-regulating microRNA-29a-3p expression. Eur Rev Med Pharmacol Sci 2021;25:1837-44.
32. Jiang R, Zhang H, Zhou J, et al. Inhibition of long noncoding RNA XIST upregulates microRNA-149-3p to repress ovarian cancer cell progression. Cell Death Dis 2021;12:145.

33. Wang S, Weng W, Chen T, et al. LINC00152 Promotes Tumor Progression and Predicts Poor Prognosis by Stabilizing BCL6 From Degradation in the Epithelial Ovarian Cancer. Front Oncol 2020;10:555132.

34. Park SA, Kim LK, Kim YT, et al. Long Noncoding RNA E2F4as Promotes Progression and Predicts Patient Prognosis in Human Ovarian Cancer. Cancers (Basel) 2020;12:3626.

35. Meng C, Zhou JQ, Liao YS. Autophagy-related long noncoding RNA signature for ovarian cancer. J Int Med Res 2020;48:300060520970761.

36. Liang H, Bai Y, Wang H, et al. Identification of LncRNA Prognostic Markers for Ovarian Cancer by Integration of Co-expression and CeRNA Network. Front Genet 2020;11:566497.

37. Li Q, Li B, Lu CL, et al. LncRNA LINC01857 promotes cell growth and diminishes apoptosis via PI3K/mTOR pathway and EMT process by regulating miR-141-3p/ MAP4K4 axis in diffuse large B-cell lymphoma. Cancer Gene Ther 2021;28:1046-57.

38. Chen GR, Sun W, Zheng K, et al. LINC01857 promotes the development of gastric cancer by regulating microRNA-200b. Eur Rev Med Pharmacol Sci 2020;24:3648-56.

39. $\mathrm{Hu}$ G, Liu N, Wang H, et al. LncRNA LINC01857 promotes growth, migration, and invasion of glioma by modulating miR-1281/TRIM65 axis. J Cell Physiol 2019;234:22009-16.

40. Xiong Y, Gu Y, Wang F, et al. LINC01857 as an oncogene regulates CREB1 activation by interacting with CREBBP in breast cancer. J Cell Physiol 2019;234:14031-9.

41. Yang Y, Dai W, Sun Y, et al. Long non-coding RNA linc00239 promotes malignant behaviors and chemoresistance against doxorubicin partially via activation of the PI3K/Akt/mTOR pathway in acute myeloid leukaemia cells. Oncol Rep 2019;41:2311-20.

42. Luo X, Yue M, Li C, et al. Long Non-Coding RNA LINC00239 Functions as a Competitive Endogenous RNA by Sponging microRNA-484 and Enhancing KLF12 Expression to Promote the Oncogenicity of Colorectal Cancer. Onco Targets Ther 2020;13:12067-81.

43. Biankin AV, Waddell N, Kassahn KS, et al. Pancreatic cancer genomes reveal aberrations in axon guidance pathway genes. Nature 2012;491:399-405. 
44. Cai T, Long J, Wang H, et al. Identification and characterization of miR-96, a potential biomarker of NSCLC, through bioinformatic analysis. Oncol Rep 2017;38:1213-23.

Cite this article as: Peng $\mathrm{J}$, Hao Y, Rao B, Zhang Z. A ferroptosis-related lncRNA signature predicts prognosis in ovarian cancer patients. Transl Cancer Res 2021;10(11):4802-4816. doi: $10.21037 /$ tcr-21-1152
45. Jurcak NR, Rucki AA, Muth S, et al. Axon Guidance Molecules Promote Perineural Invasion and Metastasis of Orthotopic Pancreatic Tumors in Mice. Gastroenterology 2019;157:838-850.e6. 